\title{
Evaluation of the Prevalence of Otomycosis in Patients Referred to the ENT Clinic
}

\author{
Habib Merza, Jenan Abdulkhaleq \\ ENT, Head \& Neck Surgery Department, Salmaniya Medical Complex, Ministry of Health, Manama, Kingdom of Bahrain \\ Email: drhabibmerza@yahoo.com
}

How to cite this paper: Merza, $H$. and Abdulkhaleq, J. (2021) Evaluation of the Prevalence of Otomycosis in Patients Referred to the ENT Clinic. International Journal of Otolaryngology and Head \& Neck Surgery, 10, 392-397.

https://doi.org/10.4236/ijohns.2021.105036

Received: July 26, 2021

Accepted: September 3, 2021

Published: September 6, 2021

Copyright (c) 2021 by author(s) and Scientific Research Publishing Inc. This work is licensed under the Creative Commons Attribution International License (CC BY 4.0).

http://creativecommons.org/licenses/by/4.0/

\begin{abstract}
Objective: Otomycosis is a common fungal infection of the outer ear that is seen in tropical and subtropical regions of the world. This disease is one of the common problems that ENT specialists, face. Therefore, this study was conducted to evaluate the status and frequency of this disease in patients referred to the ENT clinic of Salmaniya Medical Complex, Kingdom of Bahrain for one year (2019-2020). Materials \& Methods: Thirty-five patients (23 males and 12 females) were mycologically examined with a clinical diagnosis of otomycosis. In order to diagnose the disease, first ear sampling done and then the collected samples directly tested with $10 \% \mathrm{KOH}$ and culture of samples was performed on Saburo dextrose agar and Saburo dextrose agar containing chloramphenicol. In order to determine the fungus species, various laboratory methods and differential tests were used. Results: In this study, out of 56 patients with clinical diagnosis of otomycosis, according to laboratory results, only $38 \mathrm{pa}-$ tients $(67.8 \%)$ were diagnosed with otomycosis. Of these, 18 patients $(32.1 \%)$ were male and $20(35.7 \%)$ were female. The highest number of cases was observed in the age group of 30 - 40 years. Isolated fungal agents were: Aspergillus niger 24 cases (63.1\%), Candida albicans 9 cases (23.7\%), Aspergillus fumigatus 2 cases (5.3\%), Aspergillus glaucous 2 cases (5.3\%), and Penicillium one case (2.6\%). Conclusion: In this study, the most common fungi isolated from patients with otomycosis were Aspergillus and Candida species, which is consistent with other studies. The present study showed, otomycosis cannot be diagnosed by clinical symptoms alone and it requires laboratory diagnosis.
\end{abstract}

\section{Keywords}

Otomycosis, Aspergillus niger, Candida albicans

\section{Introduction}

Fungal spores are abundant in the respiratory air. On average, there are thou- 
sands of fungal spores per cubic meter of ambient air. Also, about 250,000 different fungal spores enter the human lung through respiration every minute [1] [2]. The presence of these spores in the air causes problems during eye, orthopedic, ear, nose and throat surgeries [3]. External ear infection is one of the most common problems faced by physicians, especially ENT specialists. External ear infection (otitis externa), inflammation of the external duct and earlobe, which is painful, in patients with immunocompromised condition can be life threatening [4] [5].

Otomycosis is a common fungal infection of the ear that is found in tropical and subtropical regions of the world. Meyer first described the fungal infection of the external ear canal in 1884 [6], which account for more than $20 \%$ of external ear infections in some parts of the world. In some cases, manipulation and cleaning of the ear by the patient can be predisposing factors [7]. Although a wide range of fungi have been reported to cause otomycosis, the most common are Aspergillus niger, Aspergillus flavus, Candida famata, Aspergillus terreus, Candida parapsilosis, Candida utilize, Rhizopus stolonifera, Candida guilliermondii, Candida krusei, Cryptococcus laurentii and Penicillium duclauxi. According to some previous studies, all cases of otomycosis are caused by Aspergillus and Candida species. Of course, Penicillium has also been mentioned as a causative factor [8] [9]. In general, this disease is significant because many physicians, particularly ENT specialists, have difficulty treating patients with otomycosis, particularly those who are immunocompromised and have been chronically ill [10]. The causes of otomycosis are mainly mycelial or mold fungi that are abundant in the environment and are transmitted to humans through dust and contaminated equipment. The purpose of this study was to investigate the relative frequency and prevalence of the disease in patients referred to the ENT clinic of Salmaniya Medical Complex.

\section{Methods \& Materials}

In this study, which was performed for one year during January 2019 and January 2020 on patients referred to the ENT clinic, 56 patients (including 26 males and 30 females) with clinical diagnosis of otomycosis were studied mycologically. Clinical diagnosis was made based on clinical signs and observation of fungal elements in the outer ear by otoscope. Ear sampling was performed with sterile swab moistened with sterile saline. Direct experiments were performed with $10 \%$ of $\mathrm{KOH}$ to observe fungal elements including hyphae, spores and germinated yeast. Samples were cultured on two media: Sabouraud dextrose agar and Sabouraud dextrose agar containing chloramphenicol $(0.05 \mathrm{mg} / \mathrm{ml})$. The inoculated plates were kept at laboratory temperature $\left(25^{\circ} \mathrm{C}\right)$ for 4 weeks for fungal growth and species determination. Fertility and morphology tests were performed on Corn Meal agar medium with Tween 80 to identify yeast species. Corn Meal Agar with Tween 80 is utilized primarily for the testing of Candida species for their ability to produce chlamydospores. Also, it can be used as a general pur- 
pose medium for the cultivation of fungi.

\section{Results}

In this study, out of 56 patients with clinical diagnosis of otomycosis, 38 (67.8\%) were diagnosed with this disease. Of these, 18 (32.1\%) were male and $20(35.7 \%)$ were female (Table 1).

The frequency distribution of patients with a diagnosis of otomycosis is shown in Figure 1. The highest number of cases was observed in the age group of $40-$ 50 years (Figure 1 ).

As shown in Table 2, the fungal agents isolated are: Aspergillus niger, 24 cases (63.1\%), Candida albicans, 9 cases (23.7\%), Aspergillus fumigatus, 2 cases (5.3\%), Aspergillus glucus, 2 cases (5.3\%) and Penicillium 1 case (2.6\%) (Table 2).

Table 1. Frequency distribution of patients with otomycosis visited ENT Clinic 2018-2019 according to the sex.

\begin{tabular}{cccc}
\hline Total & Male & Female & Total \\
\hline Number of patients & 26 & 30 & 56 \\
Number of Positive Cases & 18 & 20 & 38 \\
Positive cases in Percentage & $32.1 \%$ & $35.7 \%$ & $67.8 \%$ \\
\hline
\end{tabular}

Table 2. Frequency distribution of the fungal types isolated from positive cases.

\begin{tabular}{ccc}
\hline Type of Fungus & Number & Percentage \\
\hline Aspergillus niger & 24 & $63.1 \%$ \\
Candida albicans & 9 & $23.7 \%$ \\
Aspergillus fumigatus & 2 & $5.3 \%$ \\
Aspergillus glaucus & 2 & $5.3 \%$ \\
Penicillium & 1 & $2.6 \%$ \\
Total & 38 & $100 \%$ \\
\hline
\end{tabular}

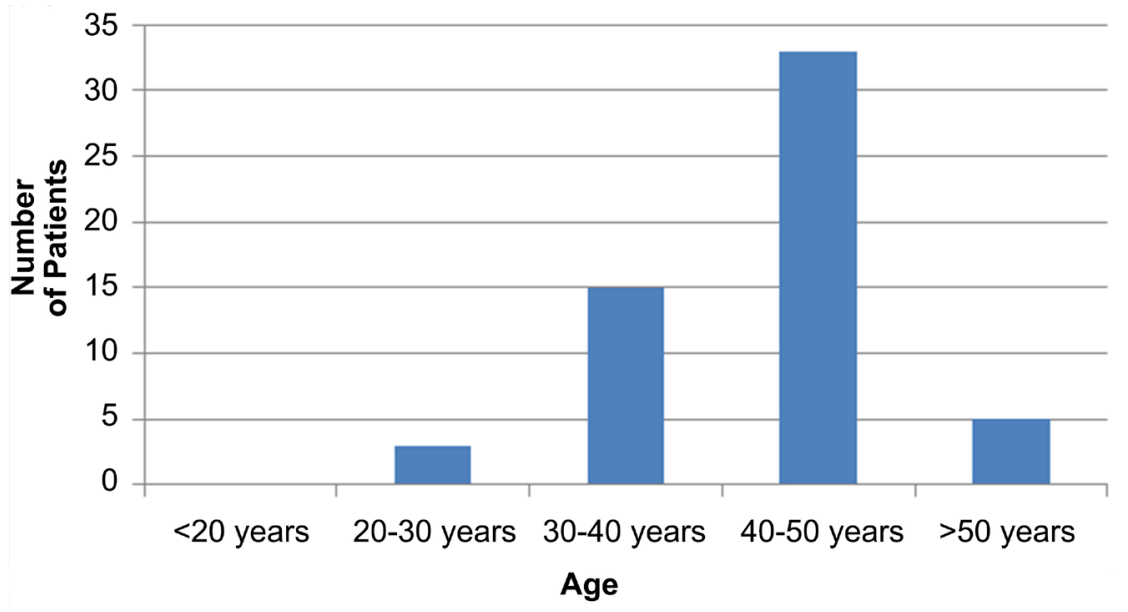

Figure 1. Frequency distribution of total number of patients with clinical diagnosis of otomycosis visited ENT clinic according to age. 


\section{Discussion}

Otomycosis is quite prevalent in the Kingdom of Bahrain as well as other countries due to temperature, humidity, and environmental dust. The disease is more common in people who work outdoors and are exposed to fungal spores. These people are mostly young and middle aged [10], which the present study also confirmed (Figure 1). Aspergillus and Candida albicans were the most common fungal species isolated from otomycosis patients in 2003 [11]. In addition, Kaur et al. reported the prevalence of Aspergillus fumigatus, Aspergillus niger, and Candida albicans in their study in 2000 [12]. The most common fungi isolated from patients in this study were Aspergillus and Candida species, which correspond with other studies. The reason for the high prevalence of Aspergillus species can be attributed to its high presence in dust and acidity of the ear canal as Aspergillus species grow more at $\mathrm{pH} 5$ to 7 [13]. In this study, Aspergillus niger was the most abundant species of Aspergillus, which is consistent with the study of other researchers [14] [15] [16] [17].

In this study, after Aspergillus species, Candida species were the most common fungi, which is consistent with the study of others [18] [19]. Protease produced by Candida species is implicated in the pathogenesis of Candida-caused otomycosis, according to research. This enzyme is involved in the colonization of Candida species on the skin and ears [20].

In the present study, the percentage of positive cases (with otomycosis) in patients with clinical symptoms of otomycosis was reported to be $67.8 \%$, which indicates that the disease cannot be diagnosed by clinical symptoms alone and requires laboratory diagnosis.

\section{Conclusion}

In ENT practice, otomycosis is a common problem. It is critical to identify the causative fungal species in order to treat cases properly. The most common fungi isolated from patients with otomycosis in this study were Aspergillus and Candida species, which is consistent with previous research. According to the findings of this study, otomycosis cannot be diagnosed based solely on clinical symptoms and requires laboratory testing.

\section{Acknowledgements}

Here we consider it necessary to thank Mr. Ali hasan, the Medical technologist in the laboratory of the Salmaniya Medical Complex, Kingdom of Bahrain, and our colleagues in the ENT clinic.

\section{Conflicts of Interest}

The authors declare no conflicts of interest regarding the publication of this paper.

\section{References}

[1] Brown, G.D., Denning, D.W., Gow, N.A.R., Levitz, S.M., Netea, M.G. and White, 
T.C. (2012) Hidden Killers: Human Fungal Infections. Science Translational Medicine, 4, 1-9. https://doi.org/10.1126/scitranslmed.3004404

[2] Sarah, C.W., Lynne, B. and Nicholas, P.M. (2016) The Fungi (Third Edition), Chapter 9-Interactions with Humans and Other Animals. Academic Press, Cambridge, 293336. https://doi.org/10.1016/B978-0-12-382034-1.00009-8

[3] Yehia, M.M., Al-habib, H.M. and Shehab, N.M. (1990) Otomycosis a Common Problem in Iraq. The Journal of Laryngology \& Otology, 104, 387-389. https://doi.org/10.1017/S0022215100158529

[4] Phillips, P., Bryce, G., Shepherd, J. and Mintz, D. (1990) Invasive External Otitis Caused by Aspergillus. Reviews of Infectious Diseases, 12, 277-281. https://doi.org/10.1093/clinids/12.2.277

[5] Mills, R. (1986) Malignant Otitis Externa. British Medical Journal, 292, 429. https://doi.org/10.1136/bmj.292.6518.429

[6] Viswanatha, B. and Naseeruddin, K. (2011) Fungal Infections of the Ear in Immunocompromised Host: A Review. Mediterranean Journal of Hematology and Infectious Diseases, 3, Article ID: e2011003. https://doi.org/10.4084/mjhid.2011.003

[7] Loh, K.S., Tan, K.K., Kumarasinghe, G., Leong, H.K. and Yeon, K.H. (1998) Otitis externa: The Clinical Pattern in a Tertiary Institution in Singapore. Annals of the Academy of Medicine of Singapore, 27, 215-218.

[8] Geaney, G.P. (1967) Tropical Otomycosis. The Journal of Laryngology \& Otology, 81, 987-997. https://doi.org/10.1017/S0022215100067955

[9] Lakshmipathi, G. and Murti, R.B. (1960) Otomycosis. Journal of Indian Medical Association, 34, 439-441.

[10] Pahwa, V.K., Chamiyal, P.C. and Suri, P.N. (1983) Mycological Study in Otomycosis. Indian Journal of Medical Research, 77, 334-338.

[11] Pradhan, B., Tuladhar, N.R. and Amatya, R.M. (2003) Prevalence of Otomycosis in out Patient Department of Otolaryngology in Tribhuvan University Teaching Hospital, Kathmandu, Nepal. Annals of Otology, Rhinology \& Laryngology, 112, 384-387. https://doi.org/10.1177/000348940311200416

[12] Kaur, R., Mittal, N., Kakkar, M.X., Aggarwal, A.K. and Mathur, M.D. (2000) Otomycosis: A Clinicomycologic Study. Ear, Nose \& Throat Journal, 79, 606-609. https://doi.org/10.1177/014556130007900815

[13] Jaiswal, S.K. (1990) Fungal Infection of Ear and It's Sensitivity Pattern. Indian Journal of Otolaryngology, 42, 19-22. https://doi.org/10.1007/BF02992530

[14] Kurnatowski, P. and Filipical, A. (2001) Otomycosis: Prevalence, Clinical Symptoms, Therapeutic Procedure. Mycoses, 44, 472-479.

https://doi.org/10.1046/j.1439-0507.2001.00689.x

[15] Ologe, F.E. and Nwabuisi, C. (2002) Treatment and Outcome of Otomycosis in Ilorin, Nigeria. West African Journal of Medicine, 21, 34-36.

[16] Mgbor, N. and Gugnani, H.C. (2001) Otomycosis in Nigeria: Treatment with Mercurochrome. Mycoses, 44, 395-397. https://doi.org/10.1046/j.0933-7407.2001.00682.x

[17] Amigot, S.L., Gomez, C.R., Luque, A.G. and Ebner, G. (2003) Microbiological Study of External Otitis in Rosario City, Argentina. Mycoses, 46, 312-315. https://doi.org/10.1046/j.1439-0507.2003.00902.x

[18] Haja, A.N., et al. (2015) Mycology of Otomycosis in a Tertiary Care Teaching Hospital. Journal of Research in Medical and Dental Science, 3, 27-30.

[19] Araiza, J., Canseco, P. and Bonifaz, A. (2006) Otomycosis: Clinical and Mycological 
Study of 97 Cases. Revue de Laryngologie Otologie, 127, 251-254.

[20] Arsenijevic, V.A., Arsovic, N. and Dzamic, A. (2004) Protease Activities of Candida SPP Isolated from Immunocompetent Patients with Otomycosis. Jugoslovenska Medicinska Biohemija, 23, Article ID: 171174.

https://doi.org/10.2298/JMH0402171A 